\title{
Dynamic tensile deformation and fracture of a highly particle-filled composite using SHPB and high-speed DIC method
}

\author{
Z. Zhou, P. Chen a , B. Guo, and F. Huang \\ State Key Laboratory of Explosion Science and Technology, Beijing Institute of Technology, Beijing 100081, \\ China
}

\begin{abstract}
In this work, various tensile tests, including Brazilian disc test (BDT), flattened Brazilian disc (FBD) test and semicircular bending (SCB) test, were carried out on a highly particle-filled composite by using a split Hopkinson pressure bar (SHPB). With the consideration of low strength and low wave impedance of the materials, a quartz crystal transducer was embedded in SHPB to measure the loading forces. A high-speed camera was used to capture the deformation and fracture process of materials. Digital image correlation (DIC) method was used to process these digital images to obtain the dynamic deformation information. Based on the measured strain fields, the crack growth path was determined and the failure mechanism of samples was analyzed. Combining SHPB and DIC method, the indirect tensile stress strain plots of disc samples were obtained, and the dynamic fracture toughness of materials was measured using both FBD and SCB tests. The results show that the tensile failure strength and fracture toughness increases with the increase of strain rates, exhibiting strain rate dependence. The high-speed DIC method combined with SHPB is effective to study the dynamic tensile behaviour of brittle materials with low strengths.
\end{abstract}

\section{Introduction}

In studying brittle solids, such as rocks, concrete and explosives, it is inconvenient to use conventional direct tensile test to measure the tensile properties of these materials due to difficulties both in specimen preparation and test operation. Some alternative methods were used to determine the tensile strength of brittle materials, such as Brazilian test [1-3], ring test [4] and bending test [5]. These methods aim at generating tensile stress in the sample by far-field compression and are easier to operate than conventional direct tensile tests.

Generally, the application of the above mentioned indirect tensile methods to the measurement of tensile strengths of brittle materials are mainly limited to quasistatic loading conditions. The measurement of tensile properties of brittle materials under impact loadings still remains challenging, primarily due to the difficulties in experimentation and data interpretation. Combining the split Hopkinson pressure Bar (SHPB) with Brazilian test and semi-circular bending test, some researchers have studied dynamic tensile behaviour of rocks and explosives [6-9].

Recently, the digital image correlation (DIC) method has been widely used in experimental mechanics as a practical and effective tool for full-field deformation measurement [10]. This technique is easy to manipulate and can provide satisfactory resolution of displacement and strain fields. Some investigations have been performed to study the fracture behavior and failure mechanism of brittle materials using DIC technique at macro and micro scale level in literatures [11-14]. The method combining the impact loading system with DIC method can be used to study the tensile deformation and fracture behavior of materials.

In this paper, the dynamic tensile properties of a highly particle-filled composite were investigated. The impact

a e-mail: pwchen@bit.edu.cn tensile experiments are conducted by means of SHPB. Three experimental methods (including Brazilian disc test, flattened Brazilian disc test and semi-circular bending test [15]) were carried out in SHPB. Because the brittle materials are of low strength and low wave impendence, application of the conventional SHPB test to measure the loading forces presents challenges, therefore, a quartz crystal transducer was embedded in the transmitted bar to measure the low amplitude loading forces. By combining the SHPB system with high-speed DIC technique, the tensile behaviour of the composite was measured.

\section{Experiments}

\subsection{Materials and specimen preparation}

The highly particle-filled composite used in this paper is a polymer-bonded explosive (PBX) simulation material, which is comprised of $\mathrm{Ba}(\mathrm{NO} 3)_{2}$ particles $(90-95 \%$ by weight) held together by fluoro-rubber ( $5-10 \%$ by weight). Molding powder was produced by a conventional slurry process, in which the inorganic particles agglomerated as they were coated with the binder dissolved in an organic solvent. The test samples were fabricated by hot isostatic pressing. The pressing pressure was $140 \mathrm{MPa}$, the pressing temperature was $100^{\circ} \mathrm{C}$ and the duration of pressing was $4 \mathrm{~h}$. Then a large piece of material ingot was obtained. The tested samples with desired geometry and size were obtained through machining. The size of disc sample was $\Phi 20 \mathrm{~mm} \times 10 \mathrm{~mm}$, the radius of SCB sample was $10 \mathrm{~mm}$ with the thickness of $10 \mathrm{~mm}$. Figure 1 displays the picture of tested samples.

\subsection{Digital image correlation (DIC) technique}

Digital image correlation (DIC) method is a non-contact optical method for displacement and strain measurement. 


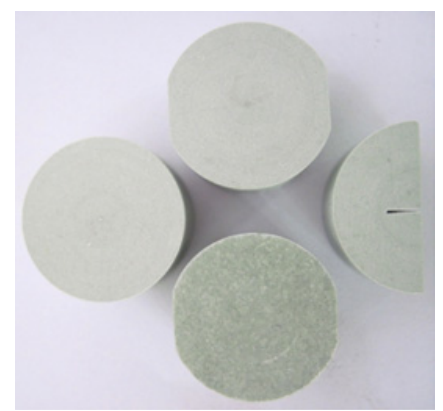

Fig. 1. Picture of the tested samples.

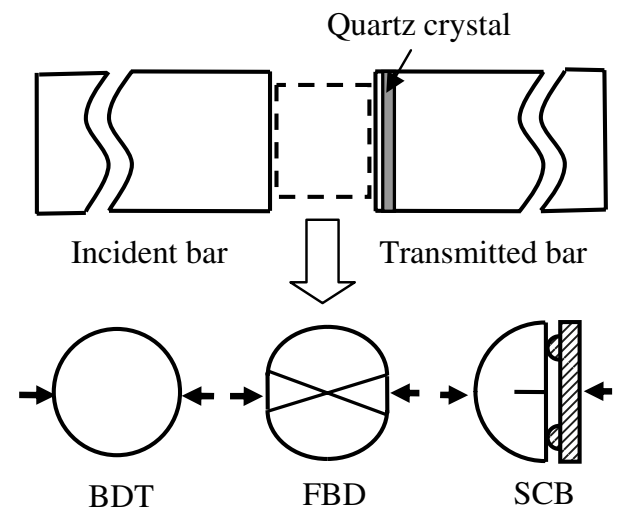

Fig. 2. Geometry of SHPB loading system.

This method utilizes a natural or artificial surface speckle pattern as an information carrier and cross-correlation function to compare images captured before and after a small deformation, to obtain the full-field in plane displacement quantitatively [17]. The basic principle of DIC method has been developed by many authors [18-20]. Due to no contacting measurement, wide measuring range and lower apparatus requirement, DIC method has been widely used in deformation measurement. In our work, the tested samples were prayed white and black paints alternatively to form the speckle pattern for displacement and strain fullfields measurement.

\subsection{Experimental setup}

In our work, a $\Phi 20 \mathrm{~mm}$ SHPB loading system was used in compression to perform tensile splitting experiments under dynamic loading. It is composed of a $200 \mathrm{~mm}$ long striker bar, a $2000 \mathrm{~mm}$ long incident bar, and a $1500 \mathrm{~mm}$ long transmitted bar, all made of aluminium. The samples were diametrically held between the incident and transmitted bars. For brittle materials with low wave impendence, most of the incident wave is reflected at the bar-specimen surface, only a few remainder wave passes through the sample. The transmitted wave signal is weak, even covered by noise. Therefore, a new measuring method was used. A quartz crystal transducer was embedded at the head of the transmitted bar to record the transmitted wave to measure the low amplitude of loading forces. The schematic of SHPB loading system was shown in figure 2.

As shown in figure 2, the BDT, FBD and SCB tests were carried out in the SHPB. The BDT was applied to measure the indirect tensile strength of brittle materials using disc specimens. Based on the elasticity theory, the two-dimensional stress field in the disc can be derived and then simplified to determine only the stress along the loading axis. The tensile stress at the disc center is given as

$$
\sigma_{t}=\frac{2 P}{\pi D B}
$$

where $P$ is the critical fracture load, $D$ is the diameter of the disc and $B$ is the thickness.

FBD and SCB tests can be used to measure the plane strain fracture toughness of brittle materials [15]. An advantage of FBD test is that it does not require a pre-notch. However, with two parallel flats introduced to the disc sample, the theoretical displacement and strain solutions for FBD are difficult to determine. Wang et al. [16] used the method combining finite element analysis with approximate analytical formulas based on mechanics of elasticity to calculate the tensile failure strength and fracture toughness of the FBD specimen. The load angle $2 \alpha \geq 20^{\circ}$ is the critical condition for a valid FBD test. After choosing the value of $2 \alpha$, the fracture toughness of the FBD specimen is calculated by

$$
K_{I C}=\frac{P_{\min }}{\sqrt{R} \cdot B} \phi\left(\frac{a}{R}\right)
$$

where $P_{\text {min }}$ is the local minimum value when the load exceeds the critical failure load. $R$ is the radius of the FBD specimen and $B$ is the thickness. $\phi$ is a coefficient which is relative to the load angle and the crack length. The $\phi$ value can be determined by finite element analysis. In this paper, the load angle $2 \alpha=30^{\circ}, \phi=0.5985$ was used.

Considering the SCB specimen geometry (as shown in figure 2), a mode-I tensile crack is induced. When the external load $P$ reaches the critical failure load $P_{q}$, the fracture toughness can be calculated from the solutions for the stress intensity factor, which is given by

$$
K_{I C}=\frac{P_{q}}{D B} \cdot Y
$$

where

$$
Y=4.47+7.4 \frac{a}{D}-106\left(\frac{a}{D}\right)^{2}+433.3\left(\frac{a}{D}\right)^{3}
$$

$P_{q}$ is the critical failure load. $D$ is the diameter of the SCB specimen, and $B$ is the thickness. $Y$ is the dimensionless stress intensity factor as a function of the dimensionless crack length, $a / D$.

In SHPB loading system, a high-speed camera was used to observe the deformation and fracture process of samples. In the test, when the striker bar impacted the incident bar, a trigger signal generated and the digital images and stress wave signals were simultaneously recorded using the high-speed camera and quartz crystal transducer, respectively. This is important for measuring the indirect tensile stress strain curves of discs.

\section{Results and discussions}

\subsection{Deformation field measurement using DIC}

Use of a high-speed camera combined with the DIC technique can determine the 2-D strain field of the sample. The 


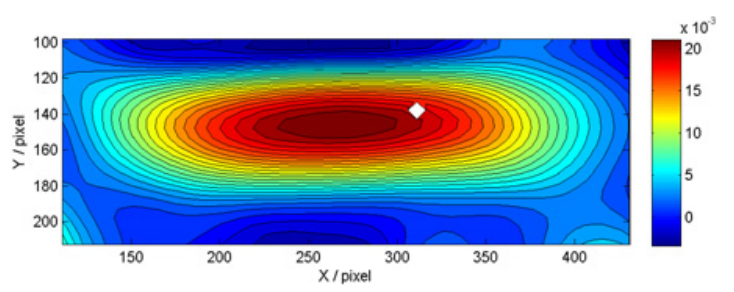

Fig. 3. Tensile strain field $\left(\varepsilon_{y y}\right)$ of disc sample prior to fracture.

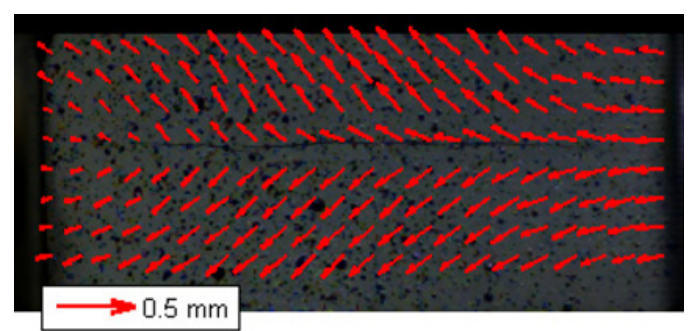

Fig. 4. Vector plots of displacement. A crack generates along the loading diameter.

frame rate of the camera was $100000 \mathrm{fps}$. Measurement was focused on the central region of the disc sample, with the image resolution of $320 \times 128$ pixels. By comparing the deformed images with initial ones, deformation fields were determined. Figure 3 shows the tensile strain field of disc sample at the moment just prior to fracturing. The result shows how the strain localizes along the loading axis. It can be seen that the strain is concentrated in the disc center with a maximum strain of approximately $2 \%$. It is believed that the crack would initiate and propagate along the loading diameter with the further increase of loading force. The vector field of displacement also gives quantitative data, indicating the deformation and failure mechanism of the sample. As shown in figure 4, the overlying arrows, shown in red, are scaled and indicate the direction of the in-plane displacements that occur from the start of the test. The vector plots of displacement clearly show that the materials above and below the failure route have vertically moved. A small crack can be seen on the sample surface. The vector plot is consistent with the strain distribution, showing that the disc sample fractures under tensile stress action. The above results are in agreement with the theoretical analysis, demonstrating the deformation localization of disc samples under impact loading.

Figure 5 shows the recorded sequence of deformation images obtained for one of the experiments on SCB specimen. Four sequential moments, A, B, C and D, were selected. The inter-frame interval was $10 \mu \mathrm{s}$. The speckle quiver plot was presented in SCB specimen surface, with both the input and output bar visible in each frame. The arrows in the figures were scaled and represented the relative degree of the displacement from the beginning of the test in each frame. At the moment $\mathrm{A}$, it is seen that the SCB sample moved towards the transmitted bar. At the following moments, a macroscopic crack initiates and propagates in the SCB sample, with tensile displacement distribution obviously perpendicular to the loading axis. Particularly, at the moment $\mathrm{D}$, the sample moved to the upper and lower sides simultaneously perpendicular to the

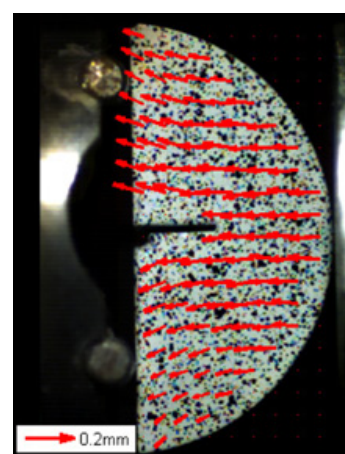

(A)

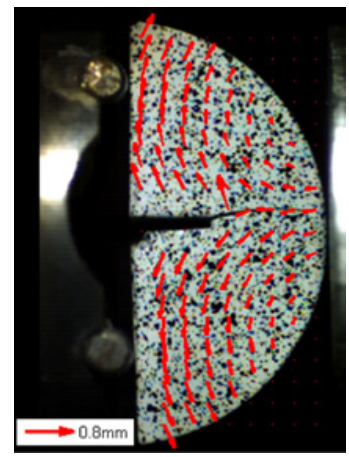

(C)

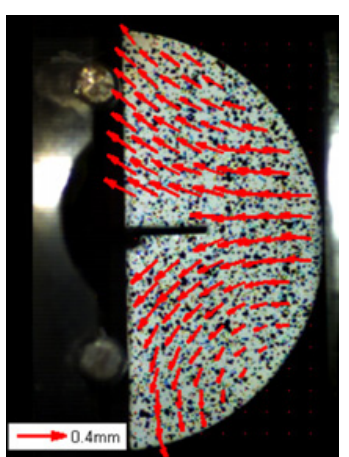

(B)

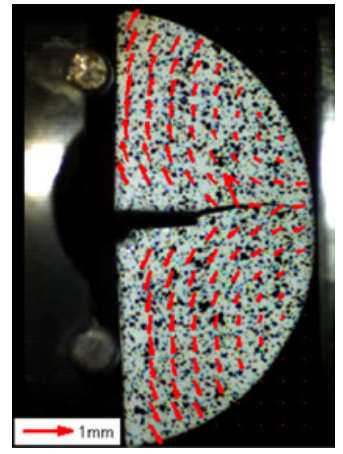

(D)
Fig. 5. Sequence of deformation images of SCB sample.

loading axis, and a macroscopic crack propagates along the pre-notch direction. The results show that the SCB sample fractures under tensile stress action subjected to high strain-rate compressive loading.

Figure 6 gives the tensile strain full-fields of SCB sample at three selected loading moments. At the moment $\mathrm{A}$, it is seen that the strain magnitude is small. In the following moments, it is seen that the localized deformation has emerged from the original per-set notch tip, demonstrated by an obvious strain concentration band generated in the head of the pre-set notch. In particular, at the moment $\mathrm{C}$, the localized deformation became more profound and the tensile strain value increases significantly with increasing of loading forces. Moreover, it is seen from figure 5(C) that a small crack generates in the local region ahead of the preset notch. The typical speckles would be broken and the local characteristics of the speckle image were changed by crack, therefore, the two local images cannot match each other between deformed and undeformed images in DIC analysis, and the displacement and strain fields of the local image cannot be calculated. Based on above analysis, the white region ahead of the pre-set notch tip in figure $6(\mathrm{C})$ can present the growth route of the crack, even though we are still unable to point the exact extent of the region occupied by the crack.

\subsection{Tensile stress strain plots measurement}

In SHPB test, the loading forces on disc samples were measured using the quartz crystal transducer. Figure 7 shows a typical load-time curve. Initially, the disc sample has a non-linear behaviour. Then the dynamic loading 


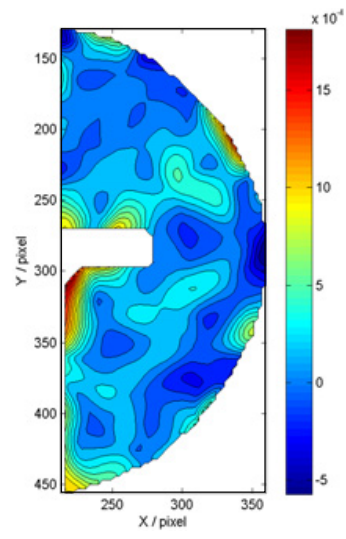

(A)

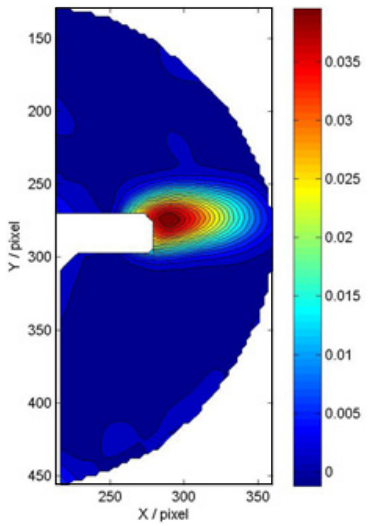

(B)

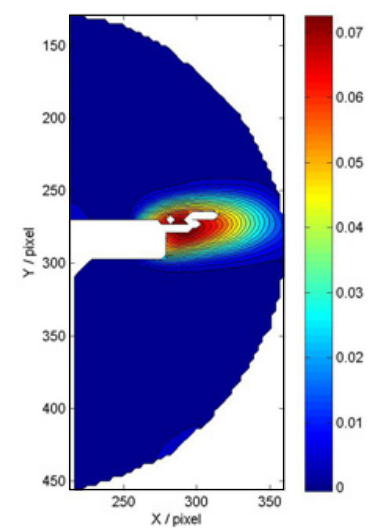

(C)

Fig. 6. Tensile strain $\left(\varepsilon_{y y}\right)$ full-fields of SCB sample at three loading moments.

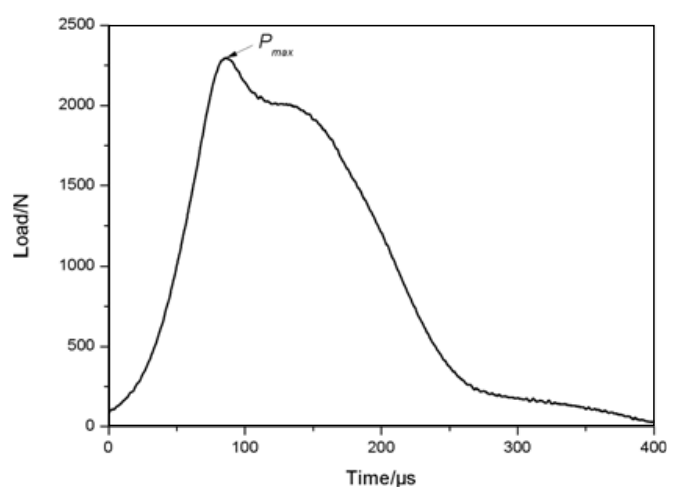

Fig. 7. Relation between dynamic loads and time of disc sample.

forces follow a period of linear increasing, and the load reaches its peak value $P_{\max }$, which corresponds to the initiation of a crack in the center of the disc sample. Then the load goes down accompanying with the crack growing quickly in the samples. The load soon reaches the 'gentle slope zone', in which the load decreases slowly. It is assumed that the crack has penetrated the loading diameter, and two separate semi-circular discs due to fracture generate. Then, the semi-circular shaped discs crushed, and the load decreased to low magnitude. Substituting the maximum load $P_{\max }$ into Eq. (1), the tensile failure strength at the disc center can be calculated.

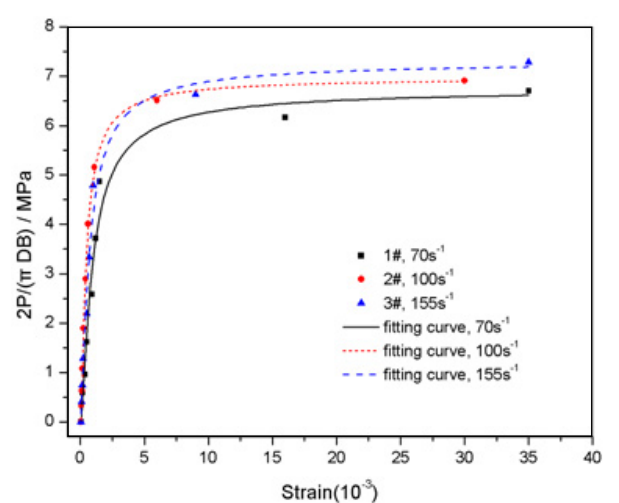

Fig. 8. Tensile stress strain plots of disc samples under different loading strain rates.

Table 1. Mechanical properties of samples under different strain rates.

\begin{tabular}{lcccc}
\hline Test & $\dot{\varepsilon} / \mathrm{s}^{-1}$ & $\sigma_{y} / \mathrm{MPa}$ & $\varepsilon / \%$ & $\mathrm{E} / \mathrm{GPa}$ \\
\hline $1 \#$ & 70 & 6.77 & 3.5 & 3.33 \\
$2 \#$ & 100 & 6.96 & 3.0 & 4.76 \\
$3 \#$ & 155 & 7.32 & 3.5 & 4.43 \\
\hline
\end{tabular}

The tensile strain fields were measured using the highspeed DIC. As discussed above (in Section 2.3), the digital images and the stress wave signals were obtained simultaneously, therefore, the tensile stresses and strains were also measured, and the indirect tensile stress strain plots at the disc center were obtained. Figure 8 shows the dynamic tensile stress strain curves of disc samples respectively, in which the dots represent the experimental data points. It is seen that the tensile failure strengths increased from 6.77 MPa to $7.32 \mathrm{MPa}$ with increasing of strain rate from $70 \mathrm{~s}^{-1}$ to $155 \mathrm{~s}^{-1}$. The result indicates that the failure stress exhibits strong strain rate dependence.

According to dynamic stress strain plots, the tensile mechanical parameters of this brittle material were calculated. Table 1 gives the tensile failure strengths, failure strains, and the elastic modulus of samples at different strain rates. The results show that the failure strain is $3.33 \pm 0.39 \%$, indicating that the failure strain remains a constant, showing little strain rate dependence. Similar studies have been performed [16], indicating that a critical strain criterion to initiate the brittle fracture of this highly particle filled composite.

\subsection{Dynamic fracture toughness measurement}

In the tests conducted using the quartz crystal technique, the relations of impact loading forces versus the loading times of FBD and SCB samples were determined. The local minimum value $P_{\min }$ and the critical fracture load $P_{q}$ can be measured. Given the geometry of FBD and SCB samples, substituting the $P_{\text {min }}$ and $P_{q}$ into Eq. (2) and Eq. (3), the dynamic fracture toughness of this composite can be calculated. The results are shown in figure 9 as triangular and dot symbols, accompanying with a linear fitting curve of these data. It is obvious that the fracture toughness increases with increasing of strain rate, showing obvious 


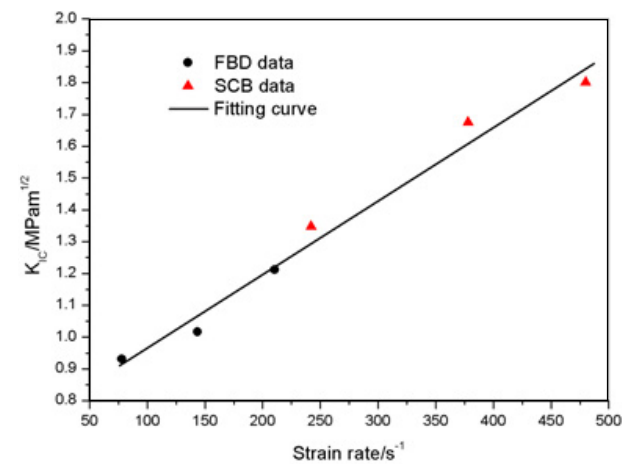

Fig. 9. Variation of dynamic fracture toughness as a function of strain rate.

strain rate dependence. Moreover, the experimental results show that the FBD and SCB tests are valid to determine the dynamic fracture toughness, exhibiting good consistency in $\mathrm{K}_{I C}$ values.

\section{Conclusions}

We used BDT, FBD and SCB tests in a SHPB system to measure the dynamic tensile deformation behaviour and mechanical properties of a highly particle-filled composite. The high-speed DIC technique was used to study the tensile deformation fields. Firstly, the dynamic displacement and strain fields were measured using DIC. The results reveal the failure mechanisms of disc samples and SCB samples. Moreover, combining the SHPB loading system with high-speed DIC technique, the tensile stress strain plots at the disc center were measured. The dynamic fracture toughness was also measured. The effects of strain rates on mechanical properties were discussed. The results show that the tensile failure strength and fracture toughness increase with increasing of strain rates, exhibiting strong strain rate dependence; however, the fracture strain remains constant, indicating a critical tensile strain criterion to the brittle fracture of this highly particlefilled composite. The results demonstrated that the method combining SHPB system with high-speed DIC technique is suitable to study the dynamic tensile behaviour of lowstrength brittle materials.

\section{References}

1. M. Mellor, I. Hawkes, Eng Geol, 5: 173-225 (1971).

2. A. Coviello, R. Lagioia, R. Nova, Rock Mechanics and Rock Engineering, 38: 251-273 (2005).

3. D. Williamson, S. Palmer, W. Proud, In proceedings: Shock Compression of Condensed Matter, 803-806 (2007).

4. J. Hudson, Int J Rock Mech Min Sci, 6: 91-97 (1969).

5. S. Dong, K. Xia, S. Huang, J Mater Sci, 46: 394-399 (2011)

6. S. Grantham, C. Siviour, W. Proud, Measurement Science and Technology, 15: 1867-1870 (2004).

7. Z. Zhou, P. Chen, S. Ma, In proceedings: 14th International Detonation Symposium (2010).

8. R. Chen, K. Xia, F. Dai, Engineering Fracture Mechanics, 76: 1268-1276 (2009).

9. Z. Zhou, P. Chen, F. Huang. In proceedings: 17th APS Shock Compression of Condensed Matter, 2011.

10. B. Pan, K. Qian, H. Xie, a review. Measurement Science and Technology, 20: 062001-7 (2009).

11. P. Rae, S. Palmer, H. Goldrein, Optics and Lasers in Engineering, 41: 635-648 (2004).

12. P. Rae, H. Goldrein, S. Palmer, In proceeding: 12th International Detonation Symposium, (2002).

13. M. Li, J. Zhang, C. Xiong, Optics and Lasers in Engineering, 43: 856-868 (2005).

14. Z. Zhou, P. Chen, F. Huang, Optics and Lasers in Engineering, 49: 366-370 (2011).

15. Z. Zhou, P. Chen, Z. Duan, F. Huang, Engineering Fracture Mechanics, 78: 2991-2997 (2011).

16. DA Wiegand, In: proceedings of 3rd Int. Conf. Def. and Frac. of Composites, 558-567 (1995).

17. C. Tay, C. Quan, Y. Huang, Optics Communications, 251: 23-36 (2005).

18. I. Yamaguchi, Proc. of SPIE, 7129, 1 9 (2008).

19. L. Yong, Q. Feng, L. Qi, Proc. of SPIE, 7160, 1-9 (2009).

20. H. Chen, D. Ye, R. Che, Proc. of SPIE, 6279, 1-7 (2007). 